\title{
Char Crystalline Transformations During Coal Combustion and Their Implications for Carbon Burnout
}

\author{
Semi-Annual Report \\ January 1 - June 30, 1997
}

\author{
By \\ Robert H. Hurt
}

Work Performed Under Contract No.: DE-FG22-95PC95205

\author{
For \\ U.S. Department of Energy \\ Office of Fossil Energy \\ Federal Energy Technology Center \\ P.O. Box 880 \\ Morgantown, West Virginia 26507-0880 \\ By \\ Division of Engineering \\ Box D \\ Brown University \\ Providence, Rhode Island 02912-9104
}




\section{Disclaimer}

This report was prepared as an account of work sponsored by an agency of the United States Government. Neither the United States Government nor any agency thereof, nor any of their employees, makes any warranty, express of implied, or assumes any legal liability or responsibility for the accuracy, completeness, or usefulness of any information, apparatus, product, or process disclosed, or represents that its use would not infringe privately owned rights. Reference herein to any specific commercial product, process, or service by trade name, trademark, manufacturer, or otherwise does not necessarily constitute or imply its endorsement, recommendation, or favoring by the United States Government or any agency thereof. The views and

opinions of authors expressed herein do not necessarily state or reflect those of the United States Government or any agency thereof. 


\section{CHAR CRYSTALLINE TRANSFORMATIONS DURING COAL COMBUSTION AND}

THEIR IMPLICATIONS FOR CARBON BURNOUT

Semi-Annual Technical Report

Reporting Period: 01/01/1997 - 06/30/1997

Author: ROBERT H. HURT

Report Issue Date: 12/30/1997

DE-FG22-95PC95205--04

ROBERT H. HURT

DIVISION OF ENGINEERING, BOX D

BROWN UNIVERSITY

PROVIDENCE, RI 02912-9104 


\section{PROJECT DESCRIPTION}

Recent work at Sandia National Laboratories, Imperial College, and the U.K. utility PowerGen, has identified an important mechanism believed to have a large influence on unburned carbon levels from pulverized coal fired boilers. That mechanism is char carbon crystalline rearrangements on subsecond times scales at temperatures of $1800-2500 \mathrm{~K}$, which lead to char deactivation in the flame zones of furnaces. The so-called thermal annealing of carbons is a well known phenomenon, but its key role in carbon burnout has only recently been appreciated, and there is a lack of quantitative data in this time/temperature range. In addition, a new fundamental tool has recently become available to study crystalline transformations, namely high resolution transmission electron microscopy (HRTEM) fringe imaging, which provides a wealth of information on the nature and degree of crystallinity in carbon materials such as coal chars.

Motivated by these new developments, this University Coal Research project has been initiated with the following two goals:

- to determine transient, high-temperature, thermal deactivation kinetics as a function of parent coal and temperature history.

- to characterize the effect of this thermal treatment on carbon crystalline structure through high-resolution transmission electron microscopy and specialized, quantitative image analysis.

Work is currently underway on the following three tasks:

\section{Task 1 Experimental technique development.}

The goal of this task is to develop and demonstrate an apparatus and procedure for measuring transient, high-temperature, thermal deactivation of coal chars. While peak gas temperatures in boilers are often in the range $1800-2000 \mathrm{~K}$, peak particle temperatures can be much higher due to high rates of heat release at the particle surface due to exothermic carbon oxidation. The prototype transient heat treatment apparatus is based on an inert-gas purged graphite-rod sample holder that is subjected to rapid Joule heating to temperatures approaching $3000{ }^{\circ} \mathrm{C}$. For the measurement of temperature histories an optical diagnostic is being developed that offers sufficient spatial resolution to distinguish the sample temperature from the substrate temperature. The optical diagnostic is based on a CID camera, a high-power lens, and movable mirrors to projecting multiple, filtered images onto a single chip. Oxidation kinetics are measured on the heat treated samples by a nonisothermal TGA technique.

\section{Task 2 Thermal deactivation kinetics.}

The goal of this task is to quantify thermal char deactivation as a function of temperature history and parent coal, with an emphasis on inert environments at temperatures and times found in combustion systems. The results are to be cast in the form of deactivation kinetics useful for incorporation in combustion models. 


\section{Task 3 Crystal structure characterization}

Crystal structure characterization provides important insight into the mechanisms of thermal char deactivation, and the degree of crystalline transformations has shown a strong correlation with reactivity changes in recent combustion studies [Davis et al., 1992, Beeley et al., 1996]. This task seeks to improve our understanding of char carbon crystalline transformations under combustion conditions by analyzing a large set of HRTEM fringe images for a series of flamegenerated chars whose reactivities have been previously reported [Hurt et al., 1995, Beeley et al., 1996]. As a first step, a new technique is being developed for the quantitative analysis of fringe images, extending previous work to allow measurement of a complete set of crystal structure parameters including mean layer size, mean stacking height, interlayer spacing, layer curvature, amorphous fraction, and degree of anisotropy. The resulting database will revealing, at a very fundamental level, the basic differences in char crystal structure due to parent coal rank and to temperature history in the range of interest to combustion systems.

\section{PROGRESS THIS PERIOD}

Progress was made this period on the development of the transient, ultra-high-temperature heat treatment apparatus for captive samples and on the development and applications of digital analysis techniques for HRTEM fringe images. The latter work focused on the quantitative characterization of orientational order in coal chars and other carbons. This work has lead to new insights into the formation mechanisms of ordered structures and is discussed in detail below.

\section{Orientational Order in HRTEM Fringe Images of Carbon}

Graphitic carbon has a highly anisotropic crystal structure with strong covalent bonding within the graphene layers and weak Van der Waals forces between layers. Many important properties of carbons are therefore directional on the microscale in a manner that depends on the local orientation of the graphene layers. In bulk samples, the length scale over which the orientational preferences are maintained (the domain size) is an important property, determining the ultimate graphitizibility of the carbon material. Carbons that possess long range orientational order $(>1 \mu \mathrm{m})$ are optically anisotropic and are capable of developing true graphitic properties upon subsequent hightemperature heat treatment. Long-range axial alignment of graphene layers is also responsible for the high tensile strength of some carbon fibers. Conversely, misalignment or the graphene layers, or of layer bundles, is responsible for the ultrafine porosity in carbons, and is believed to influence reactivity to oxidizing gases. The degree and length-scale of orientational alignment is one of the key features of carbon materials and varies greatly depending on precursor and carbonization conditions.

The goal of this work is to develop a method for quantifying orientational order in coal chars based on HRTEM fringe images. Having reduced a 002 LF image to a set of distinct fringes (as described in previous reports), the corresponding set of directional vectors can serve as a basis for useful characterizing orientational order. Figure 1 depicts simple order modes for disk-like molecules in three dimensions and their rod-like projections in two dimensions. Nematic order, a term used widely in the liquid crystal field, refers to alignment along a unique unit vector 
describing the preferential orientation of the entire field, that vector being referred to as the director. Note in Fig. 1 that the director is defined parallel to the long axis of rods, but perpendicular to the basal planes of disks by convention. Order around a point or pole in two dimensions can be concentric or radial. In three dimensions concentric order can be spherical (as in carbon blacks) or cylindrical (as in some carbon fibers). Radial order in 3D can also be spherical (though it is not typical of carbon structures) or concentric, as found in some carbon fibers. Many carbon fibers

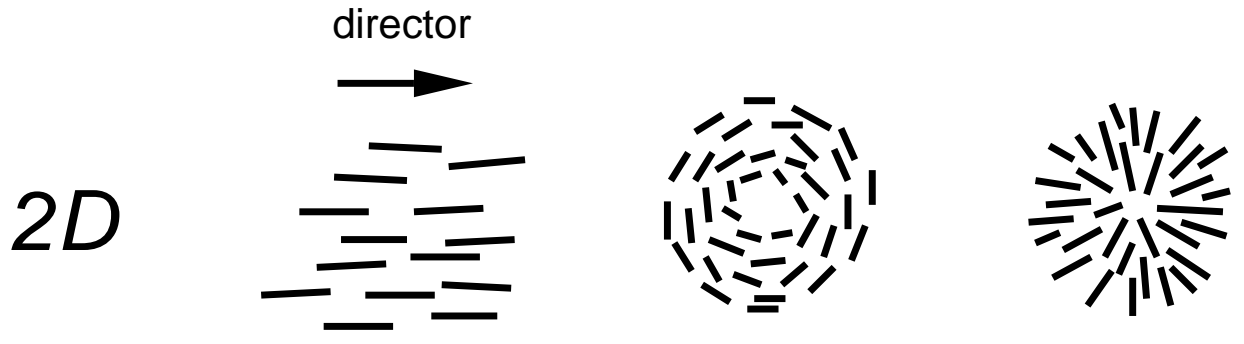

nematic circular concentric circular radial

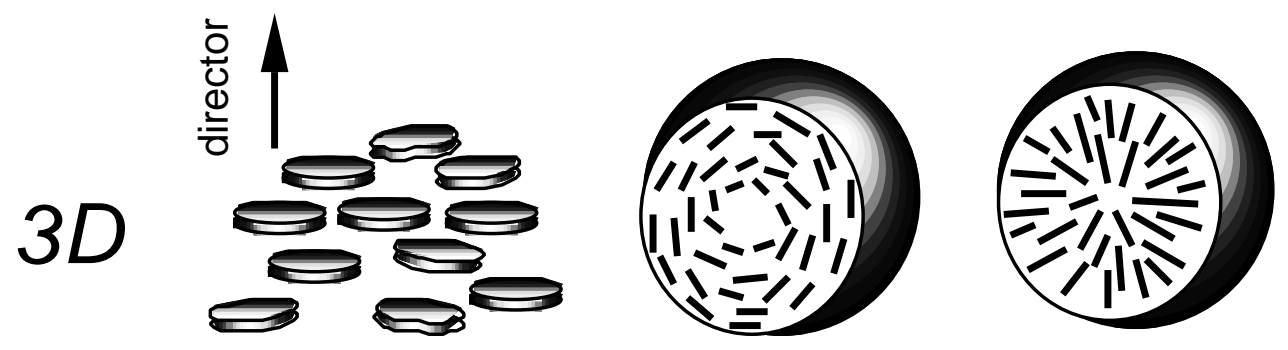

3D nematic spherical concentric spherical radial*

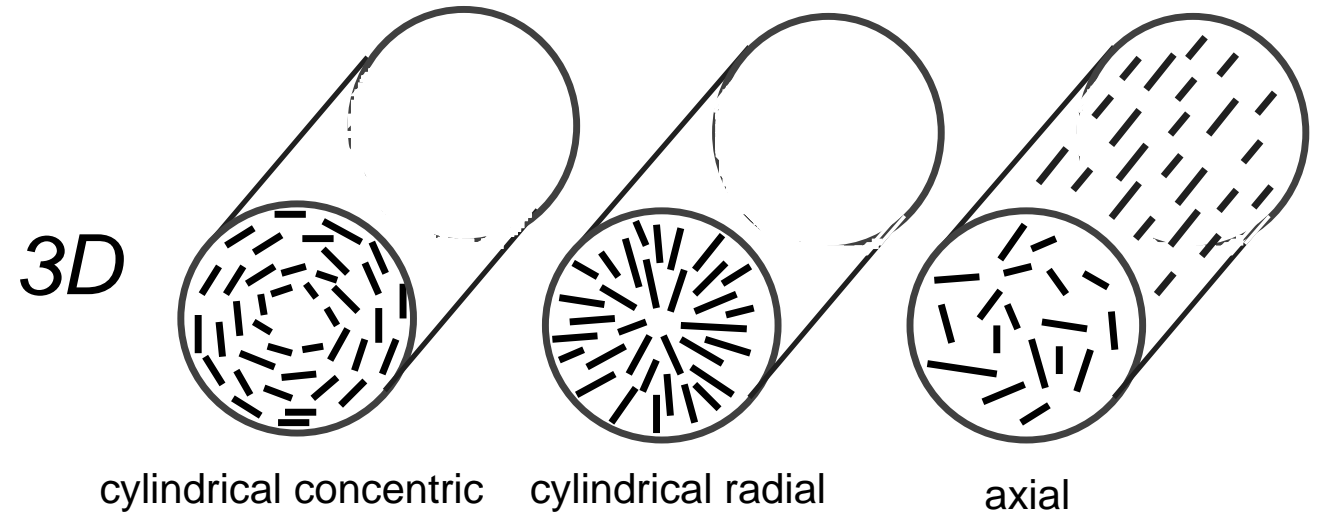

Figure 1. Modes of orientational order for disk-like objects. * There are several submodels of spherical radial order, which can be distinguished thorough additional order parameter definitions. 
exhibit axial order, or mixed modes with axial order in the core and concentric order in an outer skin [Johnson, 1985].

Equatorial stacking such as found in Brooks-Taylor mesospheres is not treated as a separate catagory, but as a nematic phase perturbed to curvature by interfacial tension. Because the relevant length scales for BT mesopheres are long (on the order of micrometers), the curvature is gradual and the underlying microscopic order is for most purposes best regarded as nematic. Equations 1 through 7 give definitions of quantitative order parameters for the various modes shown in Fig. 1. The parameters are defined to be one in the case of perfect order and zero for complete disorder (randomly aligned layers). The two dimensional parameters are most useful for the analysis of single HRTEM fringe images, while the three dimensional analogs are primarily useful in computer simulations or in reconstructions of actual structures inferred from TEM image sequences obtained upon sample rotation.

\section{Three dimensional order parameters}

The lowest orientational order mode for disk-like objects is axial orientation (see Fig. 1) in which the unit vectors all lie in a common plane, and are thus perpendicular to a unique directional vector. This type of order is seen many carbon fibers, where the preferential axial alignment induced by elongation during fiber spinning and stretching and the extent of axial alignment correlates strongly with the Youngs modulus along the fiber axis [Johnson, 1985]. In this simple mode, there is no preferred orientation of the planes when viewing along the axis. An axial order parameter in three dimensions can be defined as:

$$
S_{3 A}=1-3\left\langle\cos ^{2}\left(\theta_{i}\right)\right\rangle=1-3\left\langle\left(\overrightarrow{\mathrm{r}} \cdot \overrightarrow{\mathrm{u}}_{i}\right)^{2}\right\rangle
$$

where $\theta_{\mathrm{i}}$ is the angle between the particle axis (the $i^{\text {th }}$ directional unit vector), and the axis of symmetry, and the brackets denote an average over all objects. This order parameter is one for perfect axial order and zero for a phase with completely random alignments. A high value indicates either simple axial order or a higher degree of order: nematic, that requires an additional order parameter for its identification.

The nematic order parameter, often used to describe liquid crystalline phases, is defined by:

$$
S_{3 N}=\frac{3}{2}\left\langle\cos ^{2}\left(\theta_{i}\right)\right\rangle-\frac{1}{2}=\frac{3}{2}\left\langle\left(\overrightarrow{\mathrm{d}} \cdot \overrightarrow{\mathrm{u}}_{i}\right)^{2}\right\rangle-\frac{1}{2}
$$

where $\theta_{\mathrm{i}}$ is the angle between the axis of particle $i$, (the $i^{\text {th }}$ directional unit vector), and the mean orientation (the director). $N$ is the total number of particles, and the brackets denote an average. In the general case the director can not be found by a simple averaging procedure, but is always the eigenvector corresponding to the largest eigenvalue of the order tensor $Q$, as described in the previous quarterly. For large $\mathrm{N}$, the nematic order parameter is zero when the molecular axis are randomly distributed, and one in a system where the axis of each layer is perfectly aligned with the director. 
A polar order parameter in three dimensions, $S_{3 P}$, can be defined for both spherical and cylindrical symmetry as:

$$
S_{3 P}=\frac{3}{2}\left\langle\cos ^{2}\left(\theta_{i}\right)\right\rangle-\frac{1}{2}=\frac{3}{2}\left\langle\left(\vec{r}_{i} \cdot \vec{u}_{i}\right)^{2}\right\rangle-\frac{1}{2}
$$

For spherical symmetry $\theta \mathrm{i}$ is the angle between the orientational unit vector $i$ and the positional unit vector, $r_{i}$, that points from the origin or "pole" to the midpoint of disk $i$. The second term is an equivalent form in vector notation. This order parameter is +1 for spherical concentric and zero for disorder (limits are for large $\mathrm{N}$ ).

For cylindrical symmetry $r_{i}$ is interpreted as the position unit vector pointing from the midpoint of disk $i$ to the closest point on the cylinder axis. This order parameter is +1 for cylindrical concentric order, and $-1 / 2$ for cylindrical radial order (or for simple nematic if the director is the cylindrical axis). To distinguish cylindrical radial order from simple nematic order, one requires the following additional order parameter:

$$
S_{3 \mathrm{CR}}=\frac{3}{2}\left\langle\left(\overrightarrow{\mathrm{v}}_{\mathrm{i}} \cdot \overrightarrow{\mathrm{u}}_{i}\right)^{2}\right\rangle-\frac{1}{2} ; \text { where } \overrightarrow{\mathrm{v}}_{\mathrm{i}} \equiv \overrightarrow{\mathrm{r}}_{\mathrm{i}} \times \overrightarrow{\mathrm{a}}_{\mathrm{i}}
$$

where a new vector, $v_{i}$ is defined as the unit vector perpendicular both to the symmetry axis vector, $a_{i}$, and to the positional vector, $r_{i}$. This parameter distinguishes cylindrical radial order (where unit vectors are perpendicular to the axis) from simple nematic order (where the unit vectors are parallel to the axis), being +1 for perfect cylindrical radial order and -1 for simple nematic order.

\section{Two dimensional order parameters}

The situation in two dimensions is simpler, requiring only two order parameters, nematic and polar. Special attention is paid here to the quantitative calculation of these parameters, as they find active use in the analysis of fringe images. A two dimensional version of the nematic order parameter is defined as:

$$
S_{2 N}=2\left\langle\cos ^{2}\left(\theta_{i}\right)\right\rangle-1=2\left\langle\left(\overrightarrow{\mathrm{d}} \cdot \overrightarrow{\mathrm{u}}_{i}\right)^{2}\right\rangle-1
$$

where the director, $d$, is defined by the corresponding eigenvector from the largest eigenvalue of the ordering tensor $Q$.

This parameter is one for perfect order and, when $N$ is large, it is zero for a disordered phase with completely random orientations (e.g. isotropic carbon). Many carbon materials, particularly nongraphitizing ones, exhibit short range orientational order, but no long range order. It is desirable to be able to calculate the extent of this order as a function of length scale, which is equivalent to determining the size of the individual crystallites or molecular orientation domains. A lengthdependent nematic order parameter can be determined by selecting a series of circular image regions of varying radius, computing the order parameter for each subset of the fringes, and reporting the parameter as a function of radius. 
We have found that the traditional nematic order parameter in equation 4 is inadequate for disordered samples at small length scales, where the number of fringes present is often small $(<$ 10). For small N, even randomly distributed layers with completely uncorrelated orientations yield order parameters systematically greater than zero. This effect arises when the director is calculated from the small set of fringes themselves but disappears if the director is known a priori.. Figure 2 shows a calculation of the expected value of the conventional nematic order parameter for random lines as a function of sample size $\mathrm{N}$. For small $\mathrm{N}$ the expected value is much greater than the desired value of zero (an analytical solution to this problem was derived for $\mathrm{N}=2$ yielding $\mathrm{S}=$ $2 / \pi$, in close agreement with the numerical solution in Fig. 2.

In the practical analysis of HRTEM fringe images, the director is indeed determined from the fringe population, so a modified definition or the order parameter is needed for length-dependent calculations. The modified two-dimensional nematic order parameter is:

$$
S_{2 m N}=\left[S_{N}-F(N)\right] /[1-F(N)]
$$

where $F(N)$ is the function in Figure 2. The expected value of this parameter vanishes where no order is found and is one for a perfect alignment.

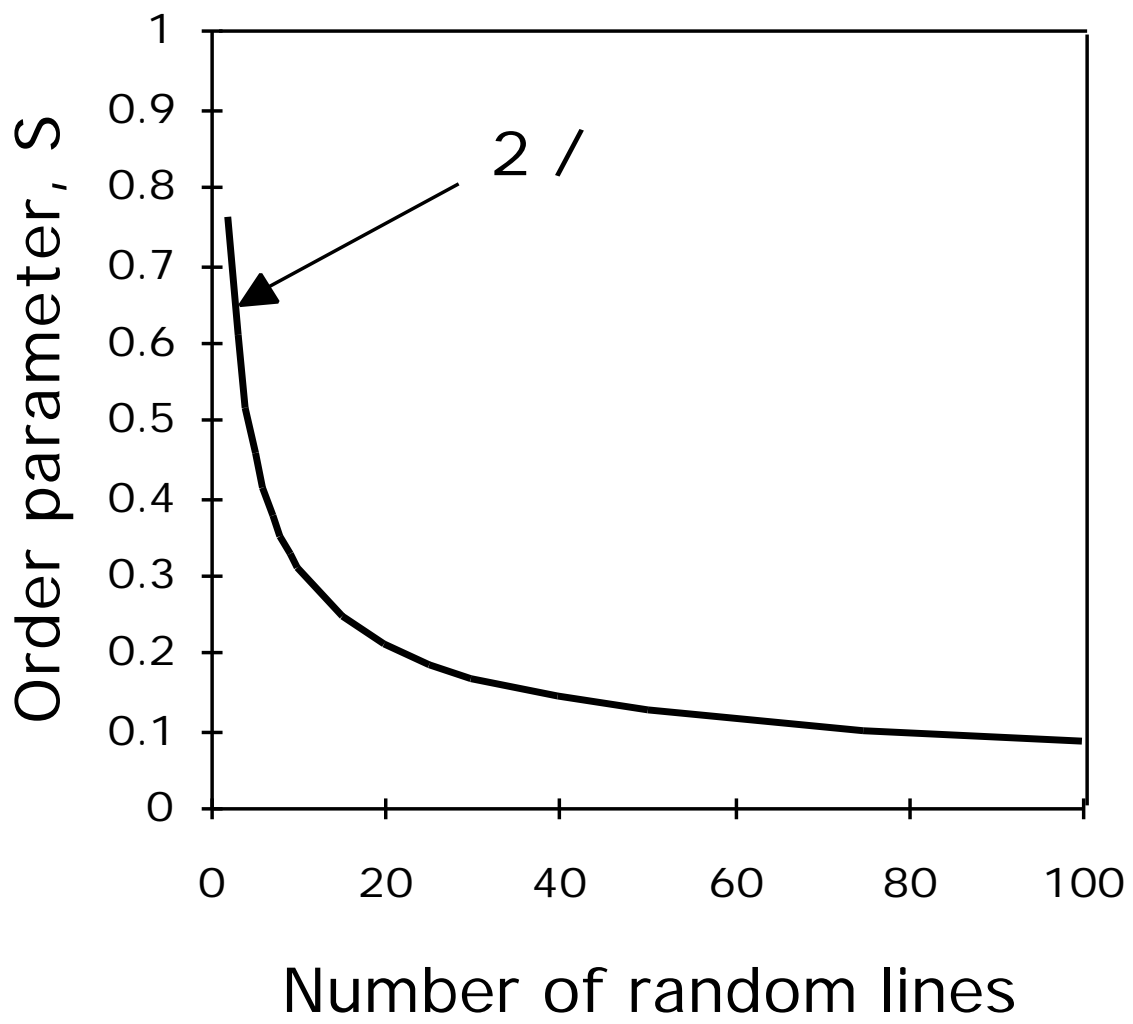

Figure 2. Pseudo-order in random lines using the conventional definition of the nematic order parameter in two dimensions. 


\section{Polar Order Parameter}

In a similar way, we define the a two-dimensional polar order parameter for fringes with either concentric or radial symmetry:

$$
S_{2 P}=2\left\langle\cos ^{2}\left(\theta_{i}\right)\right\rangle-1=2\left\langle\left(\overrightarrow{\mathrm{r}}_{\mathrm{i}} \cdot \overrightarrow{\mathrm{u}}_{i}\right)^{2}\right\rangle-1
$$

where $N$ is the total number of fringes in a circular region of interest, $u_{i}$ is the direction unit vector, and $r_{i}$ the position vector relative to the pole, which in two dimensions is always a point. A pole is always chosen prior to calculating $S_{2 P}$, so there is no need to correct the polar order parameter for statistical bias as was done for the nematic parameter. This order parameter has a value of unity for concentric symmetry, -1 for radial symmetry, and 0 for lines with random orientation.

Use of this parameter requires specification of the origin or pole. By scanning the whole image with a fixed $r_{c}$, an appropriate pole is found at the maximum value for concentric order or the minimum value for radial symmetry, and the order is calculated as a function of length with respect to the pole. The polar order parameter has been used in this laboratory to characterize concentric order in ethylene soot.

\section{Application to Coal Chars}

The previous quarterly report described our early attempts to quantify nematic orientational order in HRTEM fringe images of coal chars. An algorithm was developed to calculate the 2-D nematic order parameter from a set of skeletonized fringes in a binary image. The preprocessing techniques (Fourier-transform filtering, threshold selection, skeletonization, and reconnection) have also been discussed in previous reports. The problems associated with statistical biasing were noted, but the proper analysis of the images required the development of the modified order parameter (accomplished this period).

Figure 3 shows length-dependent nematic order parameters for four coal chars and one biomassderived char sampled from high-temperature combustion environments. The samples were collected after $72 \mathrm{msec}$ of total residence time, at which point devolatilization is complete, the peak temperatures have been reached, and the char particles are undergoing the early-to-intermediate stages of heterogeneous oxidation. The chars are seen to vary greatly in the degree and length scale of crystalline order. The biomass-derived char (switchgrass) shows a high degree of order at short length scales (below about $30 \AA$ ), but only a slight ordering tendency $(\mathrm{S}<0.25$ ) at longer length scales. This behavior is typical of samples consisting of distinct crystallites oriented nearly at random, and the length at which the order parameter decreases sharply can be used as a TEMbased definition of a "crystallite". The same general behavior is observed for the lignite and highvolatile bituminous chars, although the long-range order parameters are higher (0.5- 0.6). The Pocahontas char is unique, having a high order parameter $(S>0.9)$ that is maintained at all length scales accessible to this technique (up to $300 \AA$ ). The high degree of order and long length scale (extending across the entire HRTEM image) suggests that the order in Pocahontas was established in the fluid phase through the formation of carbonaceous mesophase, a liquid crystalline intermediate. Parallel studies are underway to characterize the optical properties of polished sections of these char samples to see if the long range order observed here is also visible under 
reflected light. We expect to see optical anisotropy for the Pocahontas representing its long range orientational order, but optical isotropy for the other samples representing the absence or weakness of long range order.

Especially noteworthy in this data set is the significant long range order in all samples. Even after correction for the apparent order in random lines, each of the samples studied retains a statistically significant non-zero order parameter up to the maximum length scale studied ( $300 \AA)$. Modified order parameters in the $0.5-0.6$ range reflect a significant alignment tendency for the three lower rank coals. This is a new finding that will likely provide new insight into the ordering mechanism and the char formation process. An important question is whether or not this moderate long-range order could arise from a liquid crystal mechanism. The next section seeks to answer this question by comparing the long-range order parameters to theoretical predictions from the Maier-Saupe mean field theory for liquid crystals.

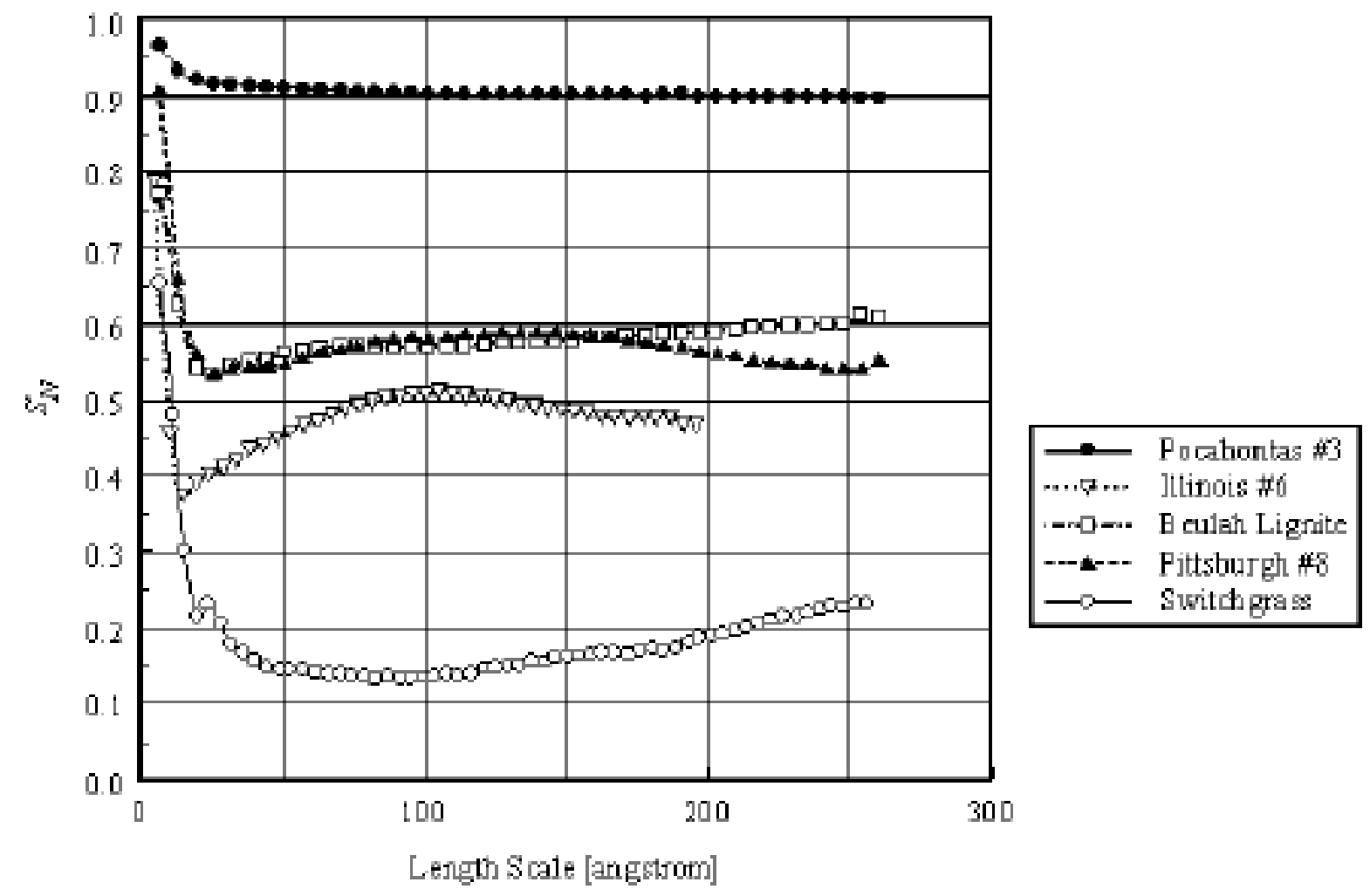

Figure 3 Modified, length-dependent, nematic order parameters for four coal chars and one biomass-derived char sampled from high-temperature combustion environments. 


\section{Mechanisms of order development in coal chars}

To better understand the mechanism leading to long-range orientational order, it will prove useful to compare the 2D order parameters to those predicted by the Maier-Saupe mean field theory for nematic liquid crystalline phases. This comparison is made difficult by the fact that fringe images are two-dimensional projections of three dimensional structures. In this section we derive the relation between the 3D and 2D order parameters and make direct comparisons between HRTEMderived order parameters and Maier-Saupe theory predictions.

Maier-Saupe mean field theory describes liquid crystal formation in terms of an anisotropic pair attractive potential of the form:

$$
\mathrm{U}(\cos \theta)=-\mathrm{v}(1 / 2)\left(3 \cos ^{2} \theta-1\right) \mathrm{S}_{3 \mathrm{~N}}
$$

where

$$
S_{3 N}=(1 / 2)\left\langle\left(3 \cos ^{2} \theta-1\right)\right\rangle
$$

The potential is designed to be zero for all molecules in the isotropic phase $\left(S_{3 N}=0\right)$ and, for ordered phases $\left(S_{3 N}>0\right)$, to vary from $+v / 2$ to $-v$ as a given molecule rotates from perpendicular to parallel with the director. The parameter $\mathrm{v}$ represents the strength of the potential. The mean field approach ignores specific pair interactions, and uses relations from classical statistical mechanics to derive the orientational distribution function and then the order parameter from Eq. 8 .

The theory gives a simple relation for the order parameter and the orientational frequency distribution in liquid crystalline phases. Figure 4 plots the predicted order parameter, $S_{3 N}$, vs. $k T / v$, showing a first order phase transition from the saturated liquid crystal phase at $S_{3 N}=0.42$ to the disordered liquid at $S_{3 N}=0$. This relation holds approximately for many liquid crystal systems and has also been shown to apply approximately to mixtures. Nematic phases are predicted to have order parameters of 0.42 or greater, the values increasing as the degree of subcooling increases. We therefore expect solid structures formed through liquid crystal intermediates to have order parameters of 0.42 or greater, a useful test for evaluating possible mechanisms of formation.

The orientational frequency distribution is given by:

$$
\rho_{3 \mathrm{D}}\left(\cos \theta_{3 \mathrm{D}}\right)=\frac{\exp \left[\mathrm{S}\left(\frac{3}{2} \cos ^{2}\left(\theta_{3 \mathrm{D}}\right)-\frac{1}{2}\right) /(\mathrm{kT} / \mathrm{v})\right]}{\int_{0}^{1} \exp \left[\mathrm{S}\left(\frac{3}{2} \cos ^{2}\left(\theta_{3 \mathrm{D}}\right)-\frac{1}{2}\right) /(\mathrm{kT} / \mathrm{v})\right] \operatorname{dcos}\left(\theta_{3 \mathrm{D}}\right)}
$$

It is this distribution which must be projected into two dimensions to compute the apparent, or two-dimensional, order parameter in a lattice fringe image. The appropriate mapping is derived in the following paragraphs. 


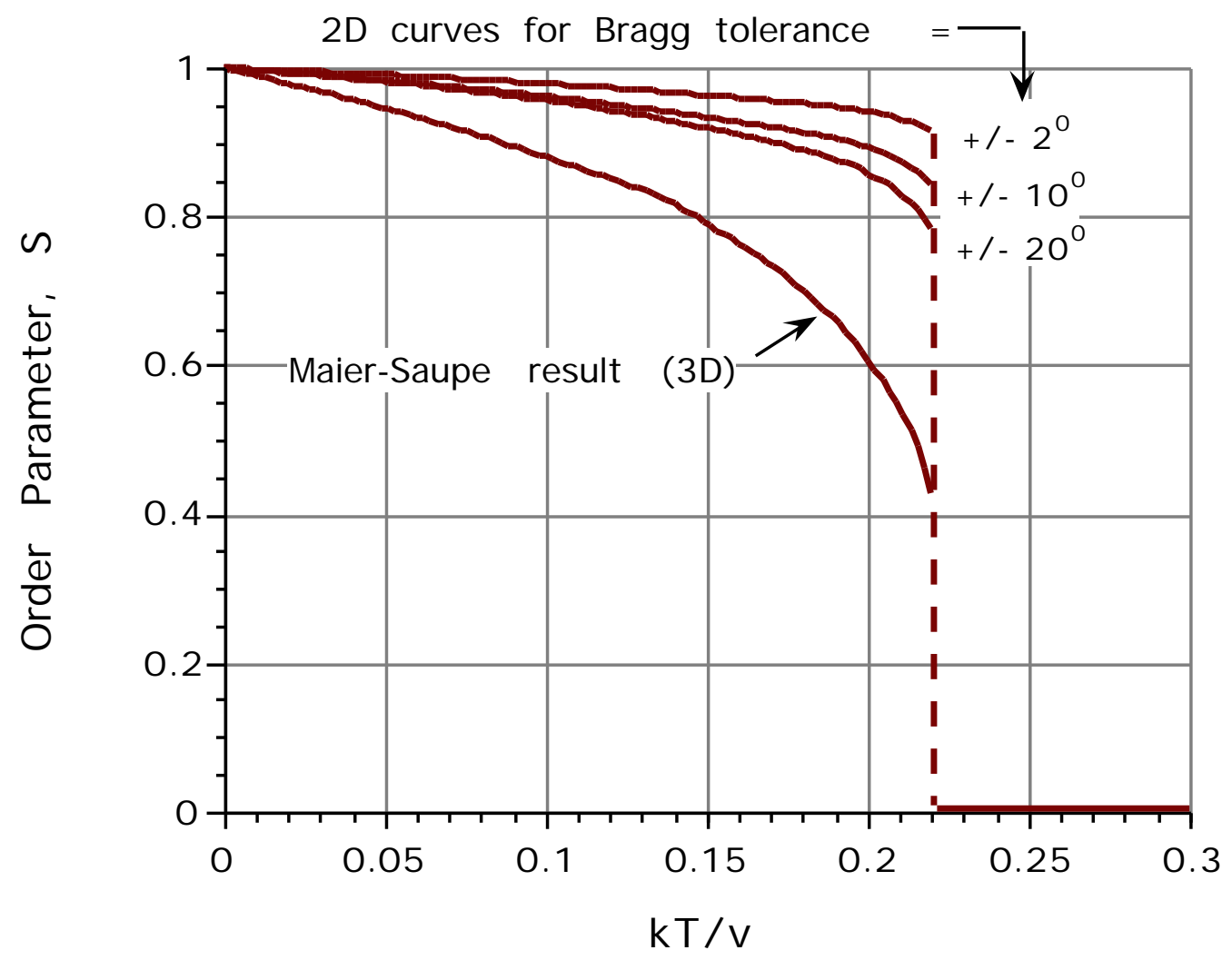

Figure 4 Three dimensional order parameters from the Maier-Saupe mean field theory and their two-dimensional counterparts relevant to the interpretation of lattice fringe images as a function of the Bragg angle tolerance.

Consider the 002 LF imaging of an anisotropic solid lattice formed by glassy solidification of a liquid crystalline phase, in which the sample tilt has been adjusted to give the maximum apparent order. The liquid crystalline director then lies in the plane perpendicular to the electron beam axis. The geometry is shown in Fig. 5, where the electron beam travels along the $\mathrm{z}$ axis, and the $\mathrm{x}$-axis is chosen for the director. Since our primary application of interest involves discotic phases, an example discotic molecule is shown along with its orientational unit vector, OA. The angle BOA represents the deviation from the director, the angle $\theta$ in equations $1-3$. The distribution is uniform in the angle, $\phi=\mathrm{CBA}$. Line segment $\mathrm{AC}$ is the perpendicular to the xy plane, and the two dimensional angle seen in a lattice fringe image is therefore BOC.

Only the layers that approximately fulfill the Bragg conditions are imaged in $002 \mathrm{LF}$, and this effect must be accounted for in the calculation of order parameters. Oberlin [1989] gives the $002 \mathrm{Bragg}$ condition as about $5 \cdot 10^{-3} \mathrm{rad}$, which is essentially zero for the purposes of this analysis. The tolerance on the Bragg angle is given by $+-\mathrm{d}_{002} / 2 \mathrm{~L}_{\mathrm{a}} \mathrm{rad}$, which is about $+/-10^{\circ}$ for $1 \mathrm{~nm}$ layers and a typical interlayer spacing [Oberlin, 1989]. If the Bragg tolerance is $+/-\alpha$, only those fringes are imaged whose directors form an angle with the xy-plane of $\alpha$ or less. On Fig. 2 that $\alpha$ is angle AOC. The Bragg tolerance must be considered here, as it effects the apparent order in two 
dimensions. One can readily see that all fringes oriented along the director $(\theta=0, \alpha=0)$ are imaged, while only a small fraction of the fringes at $\theta=\pi / 2$ will have $\alpha$ values less than $10^{\circ}$ and thus fulfill the Bragg condition within an acceptable tolerance.

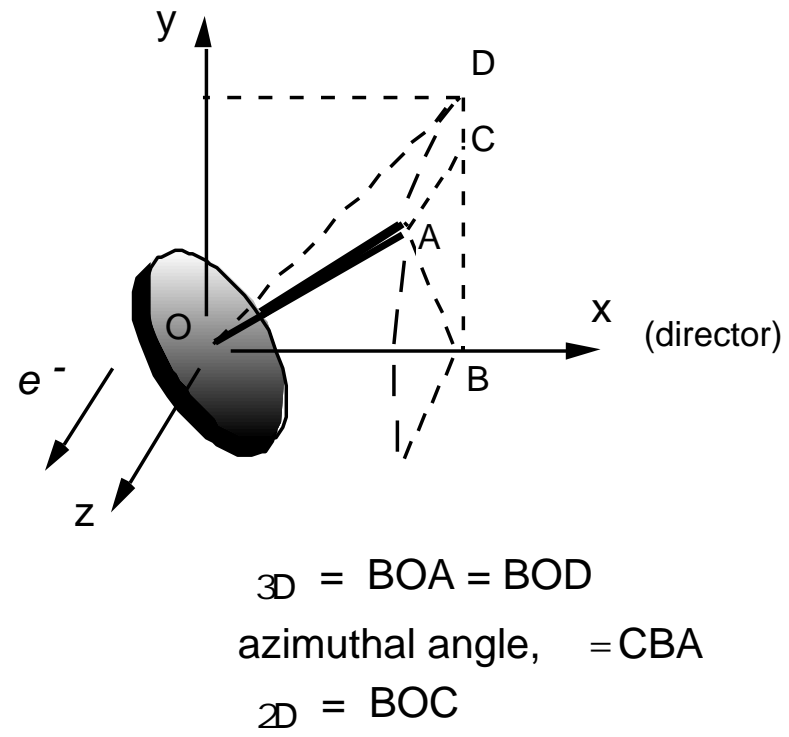

Figure 5 Nomenclature for derivation of the two-dimensional projection of three dimensional orientational order in a Maier-Saupe liquid crystalline phase. Derivation relates to imaging along the axis of maximum apparent order, where the director lies in the plane (here xy) perpendicular to the direction of travel of the electron beam (along the z-axis).

Equation 10 gives the distribution of two-dimensional angles corresponding to the Maier-Saupe orientational frequency distribution, $\rho_{3 D}\left(\cos \theta_{3 D}\right)$. Integration is performed only through those azimuthal angles, $\phi$, that lie within the Bragg tolerance, and the relation between the angle $\alpha$, on which the Bragg condition is based, and the azimuthal angle, $\phi$, is given by Eq. 11 .

$$
\rho_{2 D}\left(\cos \theta_{2 D}\right)=\int_{0}^{\phi_{\max }} \rho_{3 D}\left(\cos \theta_{3 D}\right) d \phi / \int_{0}^{1} \rho_{2 D}\left(\cos \theta_{2 D}\right) d(\cos \theta)
$$

where:

$$
\tan \left(\theta_{3 \mathrm{D}}\right)=\tan \left(\theta_{2 \mathrm{D}}\right) / \cos \phi
$$

Equations 9 - 11 were integrated for $\rho_{2 D}\left(\cos \theta_{2 D}\right)$, from which the two dimensional order parameter was computed as $2<\cos ^{2} \theta-1>$. The results are shown on Fig. 4 as a function of the Bragg angle tolerance, $+/-\alpha$. For realistic values of $\alpha$, the apparent order parameter in two 
dimensions is much higher than the order parameter in three dimensions. This is due to two effects, the more important being the fact that all of the layers oriented along the director are seen by HRTEM, while at two-dimensional angles near $\pi / 2$, a large fraction of the azimuthal distribution lies far enough off the $\mathrm{x}-\mathrm{y}$ plane to violate the Bragg criterion.

The results in Fig. 4 can be used to interpret the long-range order (10 - $200 \mathrm{~nm})$ observed in the combustion-derived coal chars. Mean values of the long range order parameters for the combustion-derived coal chars are summarized in Table 1 below. For a typical Bragg tolerance of $10^{\circ}$, Fig. 1 predicts that liquid crystal derived phases should have order parameters of 0.85 or higher. (The presence of partial columnar order, or the action of secondary rearrangements in the semi-coke stage should, if present, increase the extent of order observed in the fringe image.)

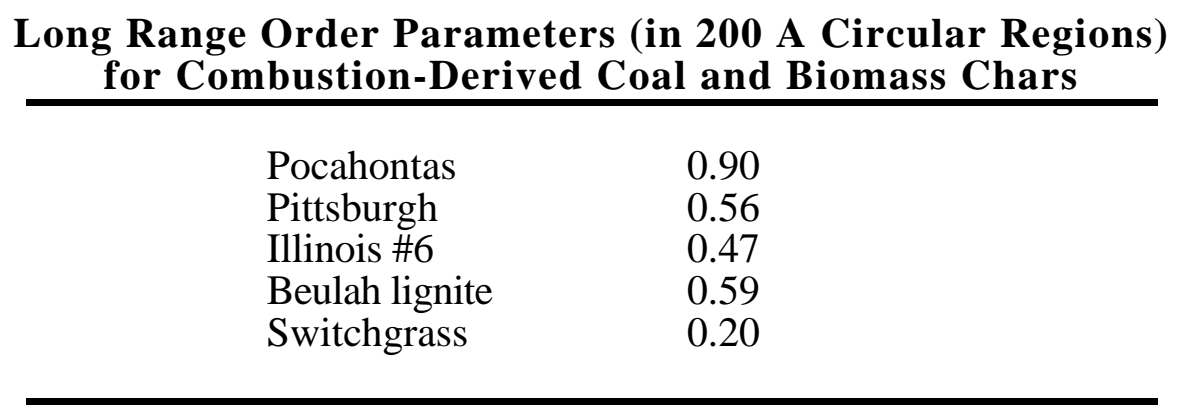

We therefore conclude that the Pocahontas char structure is quite consistent with a liquid crystal formation mechanism, but that the lower rank char structures are not. The long-range order parameters in the latter case $(0.2-0.6)$ are too low to have originated from a classical liquid crystal transition.

Based on the analysis above, we can classify the orientational order in these samples into two categories: mesophasic and statistical. Mesophasic order is the direct result of liquid crystal formation in the fluid phase followed by molecular weight growth and solidification in the ordered state to produce a structure with long range $S_{2 N}$ values greater than about 0.8 . Statistical order is characterized by lower order parameters (here below $0.2-0.6$ ) and must arise by another mechanism. There are several possible origins for the type of statistical long-range order observed in these samples. One possible origin is the anisotropy inherent in organic precursors from natural sources that is partially retained upon carbonization. Coals generally exhibit bedding plane anisotropy, and the raw plant matter is often intrinsically anisotropic due to anisometric cellular structures. NMR studies, however, have shown only a slight degree of preferential alignment of the aromatic clusters in coals below the rank of anthracite. Further, optical bireflectance (a measure of long-range anisotropy in the crystal structure) is observed only for coals of daf carbon content above about $85 \%$, represented here only by Pocahontas. Even for low-volatile bituminous coals like Pocahontas, the initial anisotropy is largely lost in the early phases of carbonization (around $400 \mathrm{C}$ ) and then reestablished, presumably by liquid crystal formation, as pyrolysis proceeds. Another mechanism is most likely responsible for the statistical long-range order seen in all these samples. 
A likely mechanism for the statistical long-range order is strain-induced alignment. Elongational flow in two-phase mixtures has been observed to induce alignment if the solid phase is composed of anisometric objects. Examples of this phenomenon are the alignment of floating logs at constrictions in rivers, the alignment of disk-like pitch components during melt-phase spinning and hot stretching of carbon fibers. Elongational strain may occur in softening coals through flow processes induced the evolution of volatile material and the growth and coalescence of bubbles. A pronounced example of this is the local stress-induced graphitization in the stretched regions adjacent to pores in non-graphitizing carbons as observed by other researchers. Local strain also occurs in the carbonization of nonsoftening coals in the form of wall stretching due to local pressure upon volatile release, or in the form of dimensional changes (shrinkage) occurring in the latter phases of mass release. Carbonization shrinkage occurring in semicokes (after initial solidification) is often observed to produce fissuring in the final coke product. It is likely that the strain-induced statistical (nonmesophasic) alignment observed in this study, if pronounced enough, leads to graphitizable regions in otherwise nongraphitizing carbons.

\section{Future Plans}

The development of digital analysis tools for high resolution TEM fringe imaging is complete. The completed tools have been applied to most of the images in the char combustion database - the remainder will be analyzed next period. Archival publications are being prepared that describe the algorithm, its application to coal char combustion, and the new insights into order development in carbons in general.

The characterization of crystal structures from heat treatment experiments and the measurement of reactivity loss with the transient, high-temperature heat treatment apparatus. Graduate student $\mathrm{H}$. Shim has completed the training necessary to carry out the highly specialized HRTEM fringe imaging technique at the Brown University facilities. The TEM technique reveals large differences between chars from different precursors (in particular from coals of varying rank) and we will continue to apply this technique to understand these differences at a very fundamental level. We will also focus on the measurement of overall reactivity loss during thermal treatment in the hightemperature heat treatment apparatus.

\section{References}

Johnson, W., Chap. 10 in Handbook of Composites, Vol. 1, Strong Fibers (Watt and Perov eds.) North-Holland, Amsterdam, 1985.

Oberlin, A. Vol 22 in Chemistry and Physics of Carbon, (P.A. Thrower, ed.), Marcel Dekker, New York, 1991. 